\title{
Editorial for rare metals, special issue on biomedical metal implants
}

\author{
Shui-Lin Wu*, Yan Li
}

Published online: 10 April 2019

(C) The Nonferrous Metals Society of China and Springer-Verlag GmbH Germany, part of Springer Nature 2019

Biomedical metal materials such as titanium, magnesium and their alloys have been widely studied as orthopedic implants or cardiovascular stents due to their desirable mechanical properties and excellent biocompatibility. However, these metal implants still exist some problems in practical application including bacterial infection, lack of bioactivity, and uncontrolled corrosion behaviors. To address these problems, the performance of these metallic implants can be improved through surface modification and incorporating alloying elements into raw materials of implants.

This special issue publishes 11 original research articles focusing on the research of biomedical metal implants. In detail, this issue covers the design of surface multifunctional coatings and composite metals with antibacterial, bioactive properties and controllable corrosion behaviors. Furthermore, the mechanical properties of porous scaffolds and new endovascular stent-graft system for endovascular aneurysm repair are also included.

Research on biomedical metal implants involves the fields of metal material science, surface science and biomedical science. This interdisciplinary filed requires indepth physical, chemical and biomedical understandings and we need to consider all things comprehensively for the fabrications of ideal biomedical metal implants. This journal will continue to offer more opportunities for publishing high-quality papers rapidly in the research of

S.-L. $\mathrm{Wu}^{*}$

School of Materials Science and Engineering, Tianjin

University, Tianjin 300072, China

e-mail: shuilinwu@tju.edu.cn biomedical metal implants and the related surface functionalization.

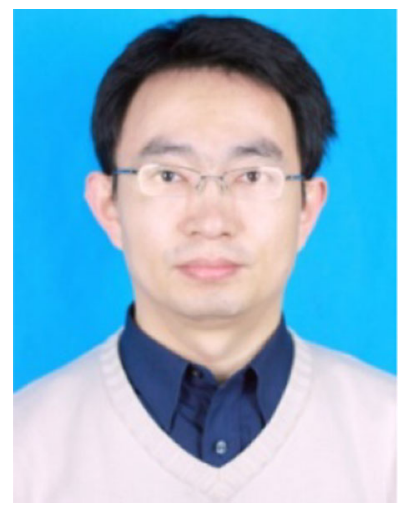

Shui-Lin Wu is a Professor of Biomaterials Science and Engineering at Tianjin University. He received his Ph.D. in 2007 from City University Hong Kong, China. He was a senior research associate and research fellow in Plasma Lab at CityU from 2007 to 2012. After that, from 2012 to 2017, he served as a professor at Hubei University. Since 2017 , He has been a faculty member in Tianjin University. His research interests involve in metallic biomaterials, surface functionalization and antibacterial system. Until now, he has published over 130 papers in peer-reviewed journals. He was also awarded as "National Science Fund for Outstanding Young Scholars" in 2014 .

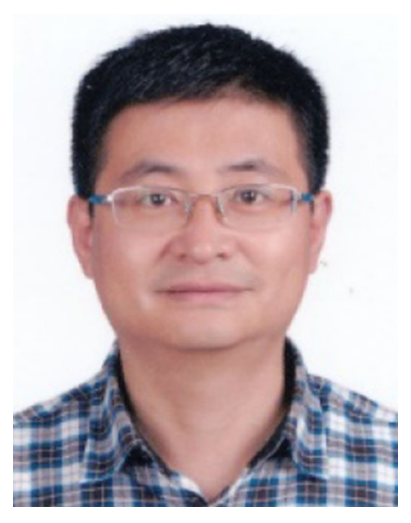

Yan Li currently a professor of the School of Materials Science and Engineering, Beihang University (BUAA). $\mathrm{He}$ received his $\mathrm{Ph} . \mathrm{D}$. degree at Dalian University of Technology in 2001, then finished the postdoctoral training at Beihang University in 2001-2003. His major research interests include biomedical materials and metal intelligent materials. Until now, he has published over 100 papers in peer-reviewed journals.
Y. Li

School of Materials Science and Engineering, Beihang University, Beijing 100191, China 